\title{
A novel approach for monitoring volatile anesthetic concentration during cardiopulmonary bypass
}

\author{
Mark M. Smith, MD · Jeffrey B. Riley, CCP • William R. Levenick, CCP • \\ Niki M. Dietz, MD
}

Received: 15 October 2015/Revised: 10 November 2015/Accepted: 13 November 2015/Published online: 3 December 2015

(c) Canadian Anesthesiologists' Society 2015

\section{To the Editor,}

Awareness and recall during general anesthesia is a rare yet serious complication that has been reported to occur in an estimated one in 8,600 cases of cardiothoracic anesthesia. ${ }^{1}$ There are numerous potential causes for awareness during cardiovascular operations. During cardiopulmonary bypass (CPB), general anesthesia is often a collaborative effort between the anesthesiologist and the perfusionist. Standard monitoring of the depth of anesthesia with end-tidal volatile concentration from the respiratory circuit is not feasible during CPB due to cessation of ventilation and lack of pulmonary blood flow. Various techniques are used to monitor the state of anesthesia during CPB, including serum volatile anesthetic concentration, end-tidal volatile anesthetic concentration at the CPB pump oxygenator exhaust line, and the bispectral index (BIS ${ }^{\mathrm{TM}}$; Minneapolis, MN, USA). ${ }^{2}$ Each of the above techniques has limitations that make universal application challenging. Analysis of serum volatile anesthetic concentration is not available for point-of-care testing, making real-time utilization impractical. Sampling end-tidal volatile anesthetic concentration at the CPB oxygenator exhaust line does not correlate well with serum plasma volatile anesthetic concentration due to the "leakiness" engineered into the membrane oxygenator. Furthermore, it can also vary with the utilization of vacuum scavenger gas systems that can further entrain room air into the exhaust line. ${ }^{3,4}$ The use of BIS during cardiac surgery is common; however, displayed

M. M. Smith, MD $(\bowtie) \cdot$ J. B. Riley, CCP .

W. R. Levenick, CCP - N. M. Dietz, MD

Department of Anesthesiology, Mayo Clinic College of

Medicine, Rochester, MN, USA

e-mail: smith.mark2@mayo.edu numerical values cannot always reliably predict the depth of anesthesia and thus far have failed to prevent awareness in large studies. ${ }^{5}$

We present a novel technique for monitoring volatile anesthetic concentration at the fresh gas inflow line prior to insertion into the membrane oxygenator. A gas sampling line with a flow restrictor connects the inflow gas line on the bypass circuit to the gas analyzer on the anesthesia machine (Figure). The inspired volatile anesthetic concentration is displayed on the anesthesia machine monitor, and low volatile alarms can be activated. Monitoring inspired volatile anesthetic concentration via this method allows for rapid detection of changes in inspired volatile anesthetic concentration, such as would occur if the vaporizer were turned off, malfunctioning, or exhausted during CPB. Unfortunately, extrapolating expiratory or "end-tidal" volatile anesthetic concentration data from the inspired concentration is not possible in patients with mechanical ventilation or during CPB. Volatile anesthetic uptake can vary during CPB depending on a number of factors, including oxygenator type and duration of usage. It can also vary in patients requiring mechanical ventilation due to ventilationperfusion (V/Q) mismatch, shunt, and fresh gas flow rate. A point to consider is that modern gas analyzers sample at a flow of $180-200 \mathrm{~mL} \cdot \mathrm{min}^{-1}$, which should not be problematic in adult patients with CPB sweep flow in excess of $2 \mathrm{~L} \cdot \mathrm{min}^{-1}$. Nevertheless, in pediatric cases, the sweep flow can be as low as $500 \mathrm{~mL} \cdot \mathrm{min}^{-1}$, risking underventilation if continuous real-time blood gas monitoring is not utilized. Again, the limitation of this sampling method is a lack of known correlation between inspired anesthetic concentration at the oxygenator inflow line and depth of anesthesia. Nevertheless, monitoring volatile concentration at the oxygenator inflow line serves 
Figure Cardiopulmonary bypass circuit with attached gas sampling line, facilitating continuous monitoring of inflow volatile anesthetic concentration at the anesthesia machine gas analyzer

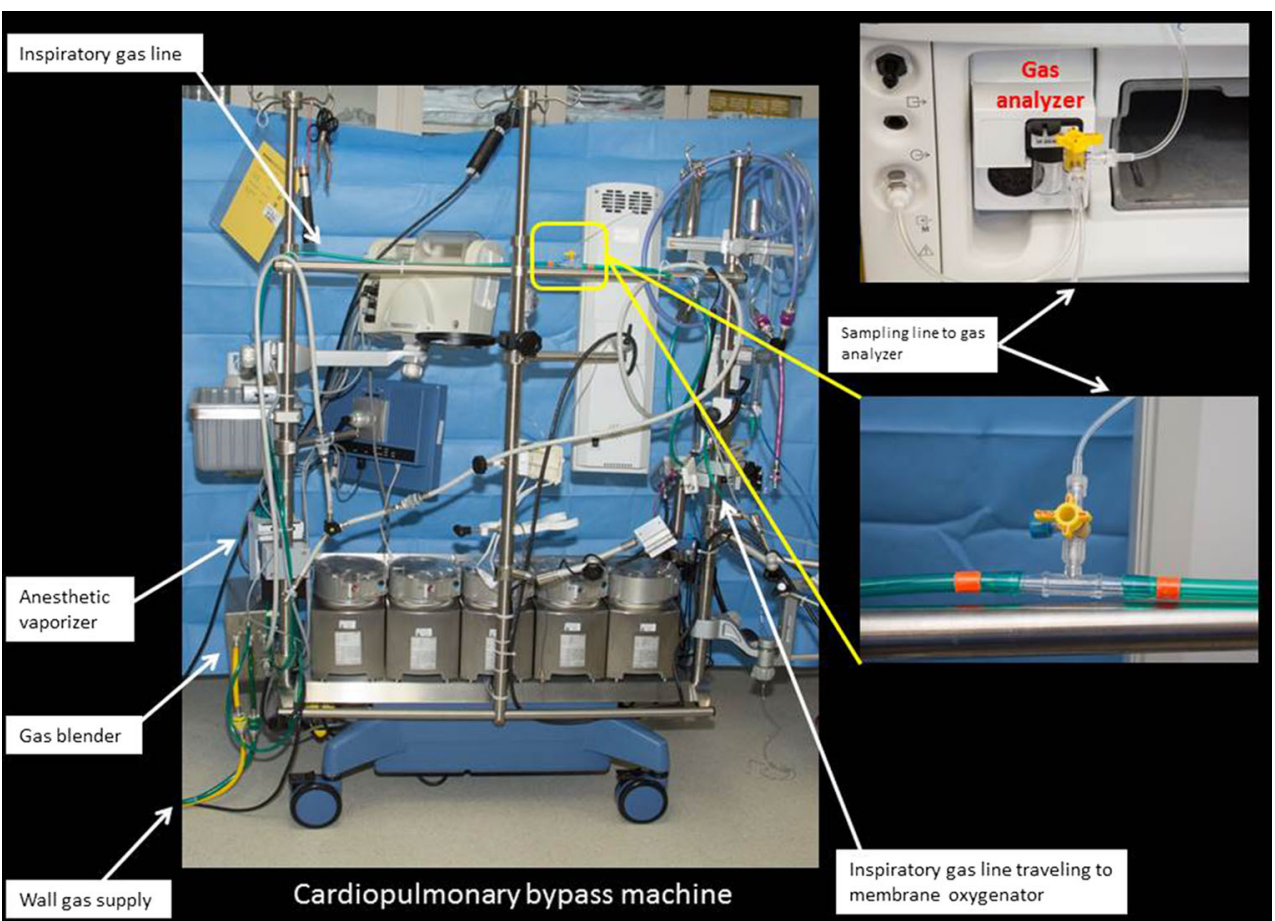

summary of main findings and risk factors. Br J Anaesth 2014; 113: 549-59.

2. Barry AE, Chaney MA, London MJ. Anesthetic management during cardiopulmonary bypass: a systematic review. Anesth Analg 2015; 120: 749-69.

3. Nitzschke R, Wilgusch J, Kersten JF, et al. Changes in sevoflurane plasma concentration with delivery through the oxygenator during on-pump cardiac surgery. Br J Anaesth 2013; 110: 957-65.

4. Homishak M, Widmer S, Stauffer R. Scavenging anesthetic gas from a membrane oxygenator during cardiopulmonary bypass. J Extra Corpor Technol 1996; 28: 88-90.

5. Avidan MS, Jacobsohn E, Glick D, et al. Prevention of intraoperative awareness in a high-risk surgical population. $\mathrm{N}$ Engl J Med 2011; 365: 591-600. 